\title{
Novel Insight Into The Potential Role of AGPAT Gene Family in Triacylglycerols Synthesis in Buffalo
}

\section{Xiaoya Ma}

Guangxi Buffalo research institute

\section{Anqin Duan}

Guangxi buffalo research institute

\section{Xingrong Lu}

Guangxi buffalo research institute

\section{Md Mahmodul Hasan Sohel}

Erciyes University

Hamdy Abdel-Shafy

Cairo University

Ahmed Amin

Cairo University

\section{Shasha Liang}

Guangxi buffalo research insititute

Tingxian Deng ( $\nabla \mathrm{dtx282000@163.com)}$

Guangxi Buffalo Research Institute https://orcid.org/0000-0002-7442-6739

\section{Research}

Keywords: AGPAT1, AGPAT6, buffalo, functional characterization, mammary epithelial cells

Posted Date: April 6th, 2021

DOI: https://doi.org/10.21203/rs.3.rs-382481/v1

License: (c) (i) This work is licensed under a Creative Commons Attribution 4.0 International License.

Read Full License 


\section{Abstract}

Background: Acylglycerophosphate acyltransferases (AGPATs) are enzymes known to act on the second acylation step in the Kennedy (de novo) pathway to catalyze the conversion of lysophosphatidic acid (LPA) to phosphatidic acid (PA). Although AGPATs have been extensively explored by evolution, expression, and functional studies, little is known about the functional characterization of how many members of the AGPAT family are involved in the triacylglycerols (TAG) synthesis. Therefore, the objective of this study was to characterize the potential functional roles of AGPAT family members in the TAG synthesis and growth of mammary epithelial cells using comparative genomic analysis and RNA interference (RNAi) technology.

Results: In the present study, a total of 13 AGPAT genes in buffalo were identified using genome-wide analysis. Among them, 12 AGPAT gene pairs were orthologous between buffalo and cattle. The comparative transcriptomic analysis and real-time quantitative reverse transcription PCR (qRT-PCR) further showed that both AGPAT1 and AGPAT6 were highly expressed in milk samples of buffalo and cattle during lactation. Notably, knockdown of AGPAT1 or AGPAT6 significantly decreased the TAG content of buffalo mammary epithelial cells (BuMEC) and bovine mammary epithelial cells (BoMEC) through regulating the mRNA expression levels of several lipogenic genes. Furthermore, the knockdown of AGPAT1 or AGPAT6 inhibited proliferation and promoted apoptosis of BuMECs; the qRT-PCR analysis of marker genes associated with cell proliferation and apoptosis supported the same conclusions.

Conclusions: These findings provide new insights about the members of AGPAT family on how they regulate the TAG synthesis and growth of mammary epithelial cells in buffalo. These findings will have important implications for understanding the role of AGPAT gene for buffalo milk production performance.

\section{Introduction}

Water buffalo (Bubalus bubalis) is important dairy livestock producing more than $15 \%$ of the world's total milk production [1]. Buffalo milk is a highly nutritious food containing $89 \%$ more fat, $28 \%$ more protein, and $100 \%$ more iron as well as $1 \%$ less lactose, and $77 \%$ less cholesterol content compared to those in dairy cattle [2]. Milk fat is composed of $98 \%$ triacylglycerols (TAG), which are the most abundant lipids in humans and animals. It is well-reported that the TAG determines the functional and physical properties of several dairy products such as the spreadability of butter [3]. The TAG is formed by a combination between glycerol and three fatty acids. The availability of fatty acids presents in milk epithelial cells along with the enzymes involved in the metabolism is largely affecting the characteristics of TAG, which is partially controlled by several genetic factors $[4,5]$. The synthesis of TAG in eukaryotes is controlled by two major pathways: the Kennedy pathway (de novo pathway) and monoacylglycerol pathway. Most TAG synthesis is performed via the de novo pathway in most cells. Evidence showed that four rate-limiting steps play a vital role in determining the triglyceride content [6], which are controlled by the glycerophosphate acyltransferase (GPAT), acylglycerophosphate acyltransferase (AGPAT), lipid 
phosphate phosphohydrolase (LPIN), and diacylglycerol acyltransferase (DGAT) gene families, respectively [7].

AGPAT family acts on the second acylation step in the de novo pathway to catalyze the conversion of lysophosphatidic acid (LPA) to phosphatidic acid (PA). To date, genome-wide identification, evolution, gene structure, and expression profile analyses have identified eleven gene members (AGPAT1-11) of the AGPAT gene family in different species [8-10]. Evidence revealed that the AGAPT family was ancient and experienced different origins, with many eukaryotic species having multiple genes originated by duplication events [8]. For example, the duplication events resulted in the origination of two isoforms AGPAT3 and AGPAT4 in animal species. Gene structure revealed that the number of introns per gene varied from one to thirteen [11]. In addition, four acyltransferase motifs (motifs I IV) were also found in the animal species. Among them, motifs I and IV contain a conserved NHxxxxD and proline (P) sequence, respectively, involving in the acyl-CoA binding and catalysis, whereas motifs II and III are involved in LPA binding, with a conserved arginine $(R)$ and EGTR sequence, respectively $[7,10,11]$. Moreover, numerous studies have characterized the expression profiles of the AGPAT gene family in different animals based on different datasets, such as the microarray expression data of human and mouse [8], RNA-seq data of chicken [9], and real-time quantitative PCR (qRT-PCR) data for cattle and pigs [12, 13], implying that different members had spatial and temporal specific expression pattern in different tissues. For example, AGPAT6 appears to be the most abundant AGPAT isoform in the bovine mammary gland, followed by AGPAT1 [13]. Numerous studies also found that AGPAT6 polymorphisms were highly associated with milk production traits in cattle including milk fat percentage [14], fat compositions [15], and fatty acids [16]. Notably, Nafikov et al. [17] also found that haplotypes of the AGPAT1 gene were associated with higher PUFA and linoleic acid concentrations in cattle. These findings suggested that both AGPAT1 and AGPTA6 play a vital role in the TAG synthesis. However, information on how many members of the AGPAT family involved in the TAG synthesis of mammary epithelial cells is still extremely limited.

Since milk production of dairy cows is significantly higher than that of buffaloes and the functional importance of AGPATs, it is of considerable importance to characterize the function of buffalo AGPAT members on the TAG synthesis. In addition, the identification of excellent functional genes will be of great significance to the molecular breeding practices of this species. In this regard, taking dairy cattle as a reference, the objectives of the present study were first to characterize the differential expression pattern of AGPAT gene family in both cattle and buffalo using comparative analysis; subsequently, to investigate the potential functional role of AGPAT genes on cell growth and TAG synthesis in buffalo mammary epithelial cells (BuMEC) and bovine mammary epithelial cells (BoMEC) using RNA interference (RNAi) technology.

\section{Materials And Methods}

\section{Identification and sequence analysis of buffalo AGPAT genes}


To perform the genome-wide identification of AGPAT genes in buffalo, we downloaded public genome datasets of seven representative mammals including the human (GRCh38.p12), cattle (ARS-UCD1.2), river buffalo (UOA_WB_1), goat (ARS1), sheep (Oar_rambouillet_v1.0), and horse (EquCab3.0) from the National Center for Biotechnology Information (NCBI) Genome database, as well as the swamp buffalo (accession number: GWHAAJZ00000000) deposited in the Genome Warehouse in the Beijing Institute of Genomics (BIG) Data Center. The known sequences of some AGPAT proteins were downloaded from the UniPort [18] and used to build the hidden Markov models (HMM) profile with the HMMER v3.3.1 software [19]. All AGPAT proteins were examined in the studied species by HMMER v3.3.1 software [19] with default parameters. Subsequently, these protein sequences were further used for multiple alignments and phylogeny tree construction implemented in MEGA-X [20] software. Identified AGPAT protein sequences were subjected to the ExPASy proteomics server for the molecular weight (MW) and isoelectric points (pl). The motif pattern and element annotation of AGPATs was analyzed using the MEME platform and Pfam programs, respectively. The conserved domain of buffalo AGPATs was predicted using CDD tools of NCBI. The motif pattern and conserved domains of buffalo AGPATs were visualized by TBtools v1.051 software [21].

\section{Chromosomal mapping and collinearity analysis for AGPAT family}

Chromosome locations of the AGPAT gene family in buffalo and cattle were obtained from their genome resources. Identification of orthologous AGPAT genes between buffalo and cattle was analyzed and visualized by TBtools v1.051 software [21] with one-step MCScanX command. Synonymous (Ks) / nonsynonymous $(\mathrm{Ka})$ substitution $(\mathrm{Ka} / \mathrm{Ks})$ rate for orthologous AGPAT pairs was calculated by the TBtools v1.051 software [21]. The divergence time for each orthologous gene pairs was evaluated by $T=$ $\mathrm{Ks} / 2 \lambda \times 10^{-6}$ million years ago (Mya) [22], where $\mathrm{T}$ is the absolute time of divergence, $K s$ is the synonymous substitution rate, and $\lambda$ is the clock-like rates in buffalo of $1.26 \times 10^{-8}$ [23].

\section{Comparative transcriptomic analyses for orthologous AGPAT genes}

To explore the differential expression of AGPAT gene family between buffalo and cattle, two published RNA-seq data (BioProject: PRJNA419906 and PRJNA453843) from milk samples were utilized for conducting the comparative transcriptome analyses. Overall, the quality control of raw data was performed by the Trim galore ver0.6.6 software [24]. Mapping of the cleaned data from buffalo and cattle was conducted by the HISAT ver.2.2.1 software [25], corresponding to the UOA_WB_1 and ARS-UCD1.2 version as reference genomes, respectively. The count matrix of gene or transcript was constructed by the StringTie ver.2.1.4 software [26], and Transcripts per million (TPM) values for each gene were obtained using the DESeq2 R-package [27]. Finally, the differential expression analysis of orthologous AGPATs between buffalo and cattle milk samples was performed. Clustering and generation of a heat map of TPM values for the selected genes were performed using the TBtools v1.051 software [21].

\section{Cell culture and transfection}


Both BuMECs and BoMECs were preserved in the Buffalo Research Institute laboratory. BuMECs were cultured in DMEM/F-12 medium (Thermo Fisher Scientific, USA) supplemented with $10 \%$ fetal bovine serum, $5 \mu \mathrm{g} / \mathrm{mL}$ insulin, $1 \mu \mathrm{g} / \mathrm{mL}$ progesterone, $1 \mathrm{ug} / \mathrm{mL}$ Hydrocortisone, $5 \mu \mathrm{g} / \mathrm{mL}$ prolactin (SigmaAldrich, USA), and $10 \mathrm{ng} / \mathrm{mL}$ epidermal growth factor 1 (Thermo Fisher Scientific, USA) and incubated at $37^{\circ} \mathrm{C}$ in a humidified atmosphere of $5 \% \mathrm{CO}_{2}$ level. At the same time, BoMECs were also cultured under the same cultural conditions. The siRNA fragments for AGPAT1 and AGPTA6 were designed and synthesized by GenePharma (Shanghai, China). These siRNA fragment sequences are listed in the Additional file 1: Table S1. Both BuMECs and BoMECs were either transfected with AGPATs siRNAs (siAGPAT) or negative control (NC) siRNAs using the RNAiMAX following the manufacturer's protocol (Thermo Fisher Scientific, USA), respectively.

\section{Cell viability assay}

Cell viability was detected after the knockdown of AGPAT1 or AGPAT6 using CellTiter-Glo® Reagent (Promega, USA) according to the manufacturer's instructions. Briefly, cells were seeded in a 96-well culture plate. At $60 \%$ confluency, cells were transfected. After treatments with three time-points $(24 \mathrm{~h}, 48$ $h$, and $72 \mathrm{~h}$ ), cells were then equilibrated at room temperature for $30 \mathrm{~min}$. Next, $100 \mu \mathrm{L}$ compound reagents were added to $100 \mu \mathrm{L}$ of medium containing cells, mixed for 2 min on an orbital shaker to induce cell lysis, incubated at room temperature for $10 \mathrm{~min}$ to stabilize the luminescent signal. Control wells containing medium without cells were also prepared to obtain a value for background luminescence, this was followed by luminescence measurement.

\section{Cell apoptosis assay}

Cell apoptosis assay was performed using Annexin V-EGFP Apoptosis Detection kit (Abcam, Cambridge, USA). According to the manufacturer's protocol, the cells were collected by centrifugation, resuspended in $500 \mu \mathrm{L}$ of binding buffer, added to $5 \mu \mathrm{L}$ of Annexin V-EGFP and $5 \mu \mathrm{L}$ of propidium iodide, and incubated at room temperature for $5 \mathrm{~min}$ in the dark. After that, the cell suspension was placed in a glass slide, and results were detected under the fluorescence microscope, where the cells which bound Annexin V-EGFP were stained with green in the plasma membrane, while cells that have lost membrane integrity were shown in red staining through the nucleus and a halo of green staining (EGFP) on the cell surface.

\section{Triglyceride assay}

Cellular TAG content in BuMECs and BoMECs was evaluated by using a TAG assay kit (Applygen, Beijing, China). Briefly, cells were seeded in 24 well plates and cultured until $60-70 \%$ confluency. Subsequently, the cultured cells were transfected with siRNA fragments. After $48 \mathrm{~h}$, the culture medium was removed and the cells were washed three times with PBS. The TAG level was calibrated with protein concentration determined with a BCA protein assay kit (Applygen, Beijing, China) and expressed as total TAG per cellar protein. Each experiment was performed in triplicate and repeated at least three times.

\section{Quantitative Real-Time PCR}


Total RNA was isolated and purified with PureLinkTM RNA Mini Kit (Thermo Fisher Scientific, USA). After that, the First Strand cDNA Synthesis Kit (Thermo Fisher Scientific, USA) was used to synthesize the firststrand of cDNA. A LightCycler 480 II sequence detection system instrument (Roche, Vienna, Austria) was used to quantify the transcript abundance of the selected genes. The qRT-PCR reactions were set up in 20 $\mu \mathrm{L}$ containing $10 \mu \mathrm{L}$ Power-up SYBR Green Master Mix (Thermo Fisher Scientific, USA), $2 \mu \mathrm{L}$ first-strand cDNA template, $0.3 \mu \mathrm{M}$ of forward and reverse gene-specific primers, and $7.4 \mu \mathrm{L}$ deionized $\mathrm{H}_{2} \mathrm{O}$. The

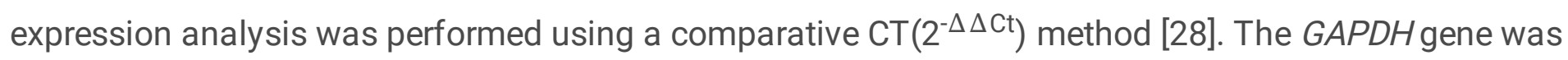

used as an endogenous control. The statistical significance between different groups was analyzed using a $t$-test and significance was declared at $P<0.05$. All the primers used for qRT-PCR are list in the Additional file 2: Table S2.

\section{Statistical analysis}

Statistical analyses were conducted using the GraphPad Prism 9.0 software [29]. The analyzed data was presented as the mean and standard error of the mean (SEM). Each test was used in triplicate. Statistical significance between the two groups was determined by the Tukey post hoc test. The significance level was declared at $P<0.05$.

\section{Results}

\section{Identification and sequence analysis of buffalo AGPAT genes}

A total of 32 and 14 AGPAT isoform protein sequences encoded by 13 AGPAT genes were predicted from the river and swamp buffalo genome using the HMMER and BLAST software, respectively (Additional file 3: Table S3). The open reading frames (ORFs) of buffalo AGPAT protein isoforms ranged from 762 to $2,136 \mathrm{bp}$ in length encoding the protein of 253 to 711 residues, with the predicted MW from 28.93 to $78.25 \mathrm{kDa}$. The pl values of these isoforms ranged from 6.20 to 11.26 .

Phylogenetic analysis revealed that all buffalo AGPAT genes could be divided into four clusters (Figure 1). Cluster IV was the top one with the larger numbers of AGPATs $(n=5)$, followed by Cluster I with 4 AGPAT genes, while Cluster II and III were the smaller ones $(n=2)$. The constructed dendrogram further showed that the buffalo AGPAT gene family was usually the most closely evolutionary relationship with the other five representative mammals (Cattle, Goat, Sheep, Horse, and Human). Moreover, the motif analysis showed that a total of 10 conserved motifs were detected in the identified buffalo AGPAT genes (Additional file 6: Figure S1). Here, 4 motifs (MEME-1, MEME-3, MEME-5, and MEME-7) were annotated as the collagen domain after the Pfam search (Additional file 4: Table S4). Interestingly, we observed that the AGPATs in Cluster I had 3 acyltransferase domains (MEME-1, MEME-3, and MEME-7) and one EF-hand_1 domain. The AGPATs in the remaining Cluster II and III had two acyltransferase domains with the MEME-1 and MEME-3 motifs, but the Cluster II AGPATs had an EF-hand_1 domain. The Cluster IV AGPATs had the MEME-3 and MEME-5 acyltransferase domains. Moreover, conserved protein domain analysis revealed that a total of 8 domains were found in the analyzed AGPAT protein sequences (Additional file 6: Figure 
S1). AGPATs in Cluster I had the LPLAT_LPCAT1-like conserved domain. The AGPATs in Cluster II and III had the acyltransferase C-terminus and LPLAT_AGPAT-like domains, respectively. The AGPATs in Cluster IV had the LPLAT_LPCAT1-like and AGPAT conserved domains.

\section{Comparative transcriptomic analyses of orthologous AGPATs between buffalo and cattle}

Prior to the comparative transcriptomic analysis of the AGPAT gene family between buffalo and cattle, we first performed collinearity analysis between the two species. The chromosomal mapping revealed that a total of 13 and 12 AGPAT genes were found to be randomly distributed on 10 chromosomes, which are mainly located on the proximate or the distal ends of the chromosomes in the buffalo and cattle, respectively (Additional file 7: Figure S2). Collinearity analysis showed that 12 AGPAT gene pairs were orthologous between the two subspecies (Figure 2A). Divergence times of all orthologous gene pairs between buffalo and cattle ranged from 0.465 to $2.937 \mathrm{Mya}$. All the orthologous gene pairs had Ka/Ks ratios less than 0.5 (Additional file 5: Table S5).

Using the RNA-seq data from the milk samples, we observed that three AGPAT genes (AGPAT1, AGPAT6, and $\angle P C A T 1$ ) were highly expressed in the three lactation stages (early-, mid-, and late-lactation) in buffalo milk (Figure 2C). By contrast, two AGPAT genes (AGPAT1 and AGPAT6) were found to be highly expressed in the same three lactation stages in cattle milk (Figure 2B). Interestingly, the expression pattern of AGPAT1 and AGPAT6 genes existed a complementation relationship in buffalo during lactation. In cattle, AGPAT6 gene was always highly expressed during lactation, followed by AGPAT1 gene. Moreover, gene expression analysis by using qRT-PCR test also showed that both AGPAT1 and AGPAT6 were highly expressed in mammary gland tissue (Figure 2D). It can be inferred that both AGPAT1 and AGPAT6 genes might have an important influence on milk production performance for both buffalo and cattle.

\section{Knockdown of AGPAT1 and AGPAT6 decreased TAG concentration in BuMEC and BoMEC}

For further exploration of the potential effect of AGPAT1 and AGPAT6 on milk performance in both buffalo and cattle, we first investigated the TGA content of BuMEC and BoMEC after the two genes silencing at $48 \mathrm{~h}$ post-transfection. As shown in Figure 3A, the interference efficiency of AGPAT1 and AGPAT6 in the BuMECs was $95 \%$ and $76 \%(P<0.0001)$, while their interference efficiency in the BoMECs was $89 \%$ and $82 \%(P<0.0001)$, respectively. The results suggested that these siRNA fragments could be used for further analysis. Subsequently, we observed that knockdown of AGPAT1 or AGPAT6 genes significantly decreased the TGA concentration in BuMECs and BoMECs (Figure 3B and 3C; $P<0.05$ ). We found that the AGPAT1 knockdown in both BuMECs and BoMECs decreased FASN, SCD, GPAM, DGAT1, $P L I N 2, A C S L 1, A C S S 1$, and LPIN1 lipogenic pathways related genes at the mRNA expression levels, while increased the expression levels of FADS2 and ACACA (Figure 3D; $P<0.05$ ). As for the knockdown of AGPAT6 gene, we observed that five lipid metabolism related genes (SCAP, FASN, FADS2, ACSL 1, and $A C A C A)$ had higher expression levels compared to the control group, while another six genes (SCD, GPAM, $D G A T 1, P L I N 2, L P I N 2$, and ACSS1) had lower mRNA expression than that of control (Figure 3E; $P<0.05$ ). 


\section{Knockdown of AGPAT1 and AGPAT6 suppress BuMECs proliferation}

The effect of AGPAT1 and AGPAT6 gene knockdown on BuMECs proliferation was investigated at three time-points $(24,48$, and $72 \mathrm{~h}$ ). Results showed that knockdown of AGPAT1 and AGPAT6 in BuMECs decreased the cell population $(P<0.05)$ in the culture medium compared to control group (Figure $4 \mathrm{~A}$ and 4C). Moreover, the mRNA levels of two proliferation-related genes (TP53 and CyclinD1) were further determined after the AGPAT1 or AGPAT6 knockdown using qRT-PCR. The results showed that increases $(P<0.05)$ in the mRNA expression level of the TP53 gene were observed after the AGPAT1 (Figure 4B) and AGPAT6 (Figure 4D) silencing. For the CyclinD1, the significant level was observed after the AGPAT1 (Figure 4B) and AGPAT6 (Figure 4D) knockdown.

\section{Knockdown of AGPAT1 and AGPAT6 promotes BuMECs apoptosis}

Using the Annexin V-EGFP Kit, we observed the apoptosis rate of the BuMECs after AGPAT1 and/or AGPAT6 gene knockdown. The results showed that the apoptosis rate was decreased in BuMECs after AGPAT1 or/and AGPAT6 silencing at $48 \mathrm{~h}$ (Figure 5A, 5B, and 5C). The mRNA expression analysis showed that both AGPAT1 and AGPAT6 had lower expression levels than that of the control group (Figure $5 \mathrm{D} ; P<0.05)$. Moreover, expression of four marker genes (BAX, FAS, BCL-2, and Caspasse6) related to apoptosis was determined using qRT-PCR in BuMECs following treatments with siAGAPT1 and siAGPAT6. The expression level of $B A X, F A S$, and $B C L-2$ was $0.765,0.430$, and 0.619 in the siAGPAT1 group, respectively, decreasing by $23.50 \%, 37.00 \%$, and $28.10 \%$ in the control group; correspondingly, the expression level of Caspasse 6 gene was 1.410 , increasing by $41.0 \%$ in the control group (Figure 5E). Similar results were observed in the siAGPAT6 group (Figure 5F) and siAGPAT1/6 group (Figure 5G).

\section{Discussion}

In the present study, a total of 13 AGPAT genes were identified in the river and swamp buffaloes. The identified AGPAT genes were unevenly distributed on the proximate or the distal ends of 10 chromosomes in buffalo. The members of the buffalo AGPAT family were divided into four clusters based on their phylogenetic relationships. Interestingly, phylogenetic classification of the buffalo AGPAT family was also supported by conserved motif and gene structure analyses. Four highly conserved motifs were observed in the AGPAT family. The members in cluster I had three acyltransferase motifs and one EFhand_1 domain. Two acyltransferase motifs were observed in Cluster II, III, and IV. The conserved protein domain analysis also supported this point. In addition, most closely related members in the same cluster of the AGPAT family harbored similar introns-exon structures (Additional file 3: Table S3).

Expression patterns of orthologous genes are often conserved and closely related to their function [3032]. In the current study, we observed a total of 12 AGPAT genes orthologous between buffalo and cattle, suggesting that they might have a similar function in both species. A previous study reported that the AGPAT6 gene was the most abundant isoform in mammary gland tissue in cattle, accounting for $60 \%$ of all AGPAT mRNA, followed by AGPAT1 (18\%) and AGPAT3 (10\%) [13]. Another study revealed that the AGPAT6 was also highly expressed in buffalo mammary gland tissues [33]. Interestingly, our data also 
showed that both AGPAT6 and AGPAT1 were highly expressed in milk samples during lactation and mammary gland tissue in buffalo. These results suggested that the two AGPAT members played a dominant role in the milk fat synthesis pathway.

To further confirm this hypothesis, we explored the potential function of AGPAT1 and AGPAT6 genes on milk fat synthesis in the BuMECs and BoMECs. Knockdown of AGPAT1 or AGPAT6 genes significantly decreased the TAG concentration in the BuMECs and BoMECs $(P<0.05)$. We observed that the TAG content in BuMECs and BoMECs decreased by approximately $60.8 \%$ and $61.40 \%$ after AGPAT1 knockdown, respectively (Figure 3B). While, the knockdown of AGPAT6 resulted in an approximately $20 \%$ and $17 \%$ decrease in TAG content of BuMECs and BoMECs, respectively (Figure $3 C$ ). The reasons for the discrepancy may be caused by the fact the AGPAT1 and AGPAT6 genes have functional differences in regulating milk fat synthesis. Our data revealed that the knockdown of AGPAT1 and AGPAT6 in both BuMECs and BoMECs significantly decreased in the mRNA expression levels of the fatty acid synthesis and desaturation-related genes (SCD), fatty acid activation-related genes (ACSS1), TAG synthesis-related genes (GPAM, DGAT1, and LPIN1), and lipid droplet formation-related gene (PLIN2). These results indicated that both AGPAT1 and AGPAT6 genes could decrease the TAG content by regulating the lipogenic genes. Moreover, we observed that four genes (SCAP, FASN, FADS2, and ACSL1) had differential mRNA expression levels in the BuMECs after AGPAT1 or AGPAT6 knockdown. They had upward trend expression levels after AGPAT6 knockdown compared to control group, which was opposite except for FADS2 in the knockdown treatment of AGPAT1. Evidence showed that the SCAP is a regulator of the transcription activity of $F A S N$ gene involved in fatty acid synthesis [34, 35], ACSL 1 plays a role in activating fatty acids destined for the TAG synthesis [36], and FADS2 plays a role in the desaturation of fatty acid synthesis [37]. More importantly, similar results were also found in the BoMECs. These results indicated that the AGPAT1 and AGPAT6 genes had functional differences in regulating the fatty acids synthesis. In other words, the AGPAT1 knockdown inhibited fatty acids synthesis, in contrast to the knockdown of AGPAT6 which promoted fatty acids synthesis. As it is well known that triacylglycerol composes one glycerol and three fatty acids, the knockdown of AGPAT6 alleviated the decrease in the TAG concentration to some extent. This explains why the reduction rate of TAG content by the treatment of AGAPT6 knockdown is lower than that of AGPAT1. In short, these findings suggested that the role of AGPAT1 and AGPAT6 genes in determining the milk fat synthesis is not only reflected in the lipogenic genes of de novo pathway but also the effect on fatty acid synthesis.

An increasing amount of experimental evidence demonstrated that the proliferation and apoptosis of mammary epithelial cells had influences on the development of mammary gland, milk secretion, and lactation [38-40]. Using the siRNA strategy, we found that both AGPAT1 and AGPAT6 knockdown could suppress the proliferation of BuMECs. Evidence showed that the stronger expression of TP53 and CyclinD1 were associated with the inhibition [41] and promotion [42] of cell proliferation, respectively. The results suggested that both AGPAT1 and AGPAT6 inhibited the BUMEC proliferation by upregulating the mRNA expression of TP53 and downregulating the CyclinD1 expression. We also observed that both AGPAT1 and AGPAT6 reduced cell viability. It is well known that the four genes (BAX, FAS, BCL-2, and Caspasse6) were associated with cell apoptosis [43-45]. Interestingly, our data revealed that the mRNA 
expression of the selected marker genes of cell apoptosis also illustrated the same results. All these results provided evidence that the AGPAT1 or AGPAT6 gene could promote the cell apoptosis of BuMECs through downregulating the $B A X, F A S$, and $B C L-2$ genes and upregulating the Caspasse 6 gene at the mRNA expression level.

\section{Conclusion}

In conclusion, we identified 13 AGPAT genes in buffalo, of which 12 orthologous gene pairs were observed between buffalo and cattle. Our work further found that both AGPAT1 and AGPAT6 were highly expressed in milk samples in buffalo and cattle during lactation. Using in vitro assay, we recognized that both AGPAT1 and AGPAT6 genes are not just regulating the TAG synthesis in mammary epithelial cells but also affecting their growth. These findings provided new insight about the members of the AGPAT family on how they regulate the TAG synthesis and growth of mammary epithelial cells in buffalo.

\section{Declarations}

\section{Acknowledgments}

We gratefully acknowledge the persons who providing help or assistance during tissue sampling. Our thanks also go to the Key Laboratory of Buffalo Genetics, Breeding and Reproduction Technology, Ministry of Agriculture in Nanning, China for the instrument support.

\section{Author contributions}

TXD conceived and designed the experiments; XYM, AQD, XRL, and SSL were responsible for cell culture, cell proliferation, apoptosis assays, data collection, and sampling; MMHS, HA, and AA contributed to data interpretation and manuscript writing; TXD and XYM analyzed the data and prepared the manuscript. All authors read and approved the final manuscript.

\section{Funding}

This work was financially supported by the Natural Science Foundation of Guangxi, China (2020GXNSFDA297032 and 2015GXNSFBA139103).

\section{Competing interests}

The authors declare that they have no competing interests.

\section{Consent for publication}

Not applicable.

\section{Availability of data and materials}


All relevant data are available within the article and its supplementary information.

\section{Ethics statement}

This study has been approved by the Ethics Committee of Buffalo Research Institute, Chinese Academy of Agricultural Sciences (BRI-CAAS), Nanning, China.

\section{Author details}

${ }^{1}$ Key Laboratory of Buffalo Genetics, Breeding and Reproduction Technology, Buffalo Research Institute, Chinese Academy of Agricultural Sciences, Nanning530001, China. ${ }^{2}$ Department of Genetics, Faculty of Veterinary Medicine, Erciyes University, Kayseri 38039, Turkey. ${ }^{3}$ Genome and Stem Cell Centre, Erciyes University, Kayseri 38039, Turkey. ${ }^{4}$ Department of Animal Production, Faculty of Agriculture, Cairo University, El-Gamma street, 12613 Giza, Egypt.

\section{References}

1. FAO. FAOSTAT database collections. In. Rome: Food and Agriculture Organization of the United Nations; 2021.

2. Barłowska J, Szwajkowska M, Litwińczuk Z, Król J. Nutritional value and technological suitability of milk from various animal species used for dairy production. Compr Rev Food Sci Food Saf. 2011;10:291-302.

3. Staniewski B, Ogrodowska D, Staniewska K, Kowalik J. The effect of triacylglycerol and fatty acid composition on the rheological properties of butter. Int Dairy J. 2021;114:104913.

4. Bionaz M, Loor JJ. Gene networks driving bovine milk fat synthesis during the lactation cycle. BMC Genom. 2008;9(1):366.

5. Tzompa-Sosa DA, van Aken GA, van Hooijdonk ACM, van Valenberg HJF. Influence of C16:0 and long-chain saturated fatty acids on normal variation of bovine milk fat triacylglycerol structure. $\mathrm{J}$ Dairy Sci. 2014;97(7):4542-51.

6. Ahmadian M, Duncan RE, Jaworski K, Sarkadi-Nagy E, Sul HS. Triacylglycerol metabolism in adipose tissue. Future Lipidol. 2007;2(2):229-37.

7. Yamashita A, Hayashi Y, Matsumoto N, Nemoto-Sasaki Y, Oka S, Tanikawa T, Sugiura T. Glycerophosphate/Acylglycerophosphate acyltransferases. Biology (Basel). 2014;3(4):801-30.

8. Körbes AP, Kulcheski FR, Margis R, Margis-Pinheiro M, Turchetto-Zolet AC. Molecular evolution of the lysophosphatidic acid acyltransferase (LPAAT) gene family. Mol Phylogenet Evol. 2016;96:55-69.

9. Yang L, Liu Z, Ou K, Wang T, Li Z, Tian Y, Wang Y, Kang X, Li H, Liu X. Evolution, dynamic expression changes and regulatory characteristics of gene families involved in the glycerophosphate pathway of triglyceride synthesis in chicken (Gallus gallus). Sci Rep. 2019;9(1):12735.

10. Takeuchi K, Reue K. Biochemistry, physiology, and genetics of GPAT, AGPAT, and lipin enzymes in triglyceride synthesis. Am J Physiol Endocrinol Metab. 2009;296(6):E1195-209. 
11. Zhukovsky MA, Filograna A, Luini A, Corda D, Valente C. The Structure and Function of Acylglycerophosphate Acyltransferase 4/ Lysophosphatidic Acid Acyltransferase Delta
(AGPAT4/LPAATS). Frontiers in Cell and Developmental Biology 2019, 7(147).

12. Lv Y, Zhang S, Guan W, Chen F, Zhang Y, Chen J, Liu Y. Metabolic transition of milk triacylglycerol synthesis in response to varying levels of palmitate in porcine mammary epithelial cells. Genes Nutr. 2018;13:18.

13. Bionaz M, Loor JJ. ACSL1, AGPAT6, FABP3, LPIN1, and SLC27A6 are the most abundant isoforms in bovine mammary tissue and their expression is affected by stage of lactation. $\mathrm{J}$ Nutr. 2008;138(6):1019-24.

14. Wang X, Wurmser C, Pausch H, Jung S, Reinhardt F, Tetens J, Thaller G, Fries R. Identification and dissection of four major QTL affecting milk fat content in the German Holstein-Friesian population. PLoS One. 2012;7(7):e40711.

15. Littlejohn MD, Tiplady K, Lopdell T, Law TA, Scott A, Harland C, Sherlock R, Henty K, Obolonkin V, Lehnert $K$, et al. Expression variants of the lipogenic AGPAT6 gene affect diverse milk composition phenotypes in Bos taurus. PLoS One. 2014;9(1):e85757.

16. Bouwman AC, Bovenhuis H, Visker MH, van Arendonk JA. Genome-wide association of milk fatty acids in Dutch dairy cattle. BMC Genet. 2011;12:43.

17. Nafikov RA, Schoonmaker JP, Korn KT, Noack K, Garrick DJ, Koehler KJ, Minick-Bormann J, Reecy JM, Spurlock DE, Beitz DC. Polymorphisms in lipogenic genes and milk fatty acid composition in Holstein dairy cattle. Genomics. 2014;104(6):572-81. Part B).

18. Consortium TU. UniProt: a worldwide hub of protein knowledge. Nucleic acids research. 2019;47(D1):D506-d515.

19. Potter SC, Luciani A, Eddy SR, Park Y, Lopez R, Finn RD. HMMER web server: 2018 update. Nucleic acids research. 2018;46(W1):W200-w204.

20. Kumar S, Stecher G, Li M, Knyaz C, Tamura K. MEGA X: Molecular Evolutionary Genetics Analysis across Computing Platforms. Molecular biology evolution. 2018;35(6):1547-9.

21. Chen C, Chen H, Zhang Y, Thomas HR, Frank MH, He Y, Xia R. TBtools: An Integrative Toolkit Developed for Interactive Analyses of Big Biological Data. Mol Plant. 2020;13(8):1194-202.

22. Lynch M, Conery JS. The evolutionary fate and consequences of duplicate genes. Science. 2000;290(5494):1151-5.

23. Sun T, Shen J, Achilli A, Chen N, Chen Q, Dang R, Zheng Z, Zhang H, Zhang X, Wang S, et al: Genomic analyses reveal distinct genetic architectures and selective pressures in buffaloes. GigaScience 2020, $9(2)$.

24. Trim Galore! http://www.bioinformatics.babraham.ac.uk/projects/trim_galore/.

25. Kim D, Paggi JM, Park C, Bennett C, Salzberg SL. Graph-based genome alignment and genotyping with HISAT2 and HISAT-genotype. Nat Biotechnol. 2019;37(8):907-15. 
26. Kovaka S, Zimin AV, Pertea GM, Razaghi R, Salzberg SL, Pertea M. Transcriptome assembly from long-read RNA-seq alignments with StringTie2. Genome Biol. 2019;20(1):278.

27. Love MI, Huber W, Anders S. Moderated estimation of fold change and dispersion for RNA-seq data with DESeq2. Genome Biol. 2014;15(12):550.

28. Livak KJ, Schmittgen TD. Analysis of relative gene expression data using real-time quantitative PCR and the 2(-Delta Delta C(T)) Method. Methods. 2001;25(4):402-8.

29. Mitteer DR, Greer BD, Randall KR, Briggs AM. Further Evaluation of Teaching Behavior Technicians to Input Data and Graph Using GraphPad Prism. Behav Anal (Wash D C). 2020;20(2):81-93.

30. Ruvinsky I, Ruvkun G. Functional tests of enhancer conservation between distantly related species. Development. 2003;130(21):5133-42.

31. Romero IG, Ruvinsky I, Gilad Y. Comparative studies of gene expression and the evolution of gene regulation. Nature reviews Genetics. 2012;13(7):505-16.

32. Martin T, Fraser HB. Comparative expression profiling reveals widespread coordinated evolution of gene expression across eukaryotes. Nat Commun. 2018;9(1):4963.

33. Song S, Huo JL, Li DL, Yuan YY, Yuan F, Miao YW. Molecular cloning, sequence characterization, and gene expression profiling of a novel water buffalo (Bubalus bubalis) gene, AGPAT6. Genet Mol Res. 2013;12(4):4116-26.

34. Han LQ, Gao TY, Yang GY, Loor JJ. Overexpression of SREBF chaperone (SCAP) enhances nuclear SREBP1 translocation to upregulate fatty acid synthase (FASN) gene expression in bovine mammary epithelial cells. J Dairy Sci. 2018;101(7):6523-31.

35. Suburu J, Shi L, Wu J, Wang S, Samuel M, Thomas MJ, Kock ND, Yang G, Kridel S, Chen YQ. Fatty acid synthase is required for mammary gland development and milk production during lactation. American journal of physiology Endocrinology metabolism. 2014;306(10):E1132-43.

36. Li LO, Ellis JM, Paich HA, Wang S, Gong N, Altshuller G, Thresher RJ, Koves TR, Watkins SM, Muoio DM, et al. Liver-specific loss of long chain acyl-CoA synthetase-1 decreases triacylglycerol synthesis and beta-oxidation and alters phospholipid fatty acid composition. J Biol Chem. 2009;284(41):27816-26.

37. Bionaz M, Loor JJ. Gene networks driving bovine milk fat synthesis during the lactation cycle. BMC Genom. 2008;9:366.

38. Capuco AV, Wood DL, Baldwin R, McLeod K, Paape MJ. Mammary cell number, proliferation, and apoptosis during a bovine lactation: relation to milk production and effect of bST. J Dairy Sci. 2001;84(10):2177-87.

39. Ji Z, He R, Chao T, Xuan R, Liu S, Wang G, Wang J: chi-miR-143-3p Promotes Apoptosis of Mammary Gland Epithelial Cells from Dairy Goats by Targeting Ndfip1. DNA Cell Bio/ 2019, 38(11):1188-1196.

40. Ji Z, Wang G, Hou L, Liu Z, Wang J, Chao T. miR-143 inhibits proliferation and induces apoptosis of mammary epithelial cells in dairy goat. Animal Cells Systems. 2016;20(2):63-9. 
41. Wawryk-Gawda E, Chylińska-Wrzos P, Lis-Sochocka M, Chłapek K, Bulak K, Jędrych M. JodłowskaJędrych B: P53 protein in proliferation, repair and apoptosis of cells. Protoplasma. 2014;251(3):52533.

42. González-Sancho JM, Figueroa A, López-Barahona M, López E, Beug H, Muñoz A. Inhibition of proliferation and expression of $\mathrm{T} 1$ and cyclin $\mathrm{D} 1$ genes by thyroid hormone in mammary epithelial cells. Mol Carcinog. 2002;34(1):25-34.

43. Liang S, Sun K, Wang Y, Dong S, Wang C, Liu L, Wu Y. Role of Cyt-C/caspases-9,3, Bax/Bcl-2 and the FAS death receptor pathway in apoptosis induced by zinc oxide nanoparticles in human aortic endothelial cells and the protective effect by alpha-lipoic acid. Chem Biol Interact. 2016;258:40-51.

44. Li J, Yuan J. Caspases in apoptosis and beyond. Oncogene. 2008;27(48):6194-206.

45. Planells-Ferrer L, Urresti J, Coccia E, Galenkamp KM, Calleja-Yagüe I, López-Soriano J, Carriba P, Barneda-Zahonero B, Segura MF, Comella JX. Fas apoptosis inhibitory molecules: more than deathreceptor antagonists in the nervous system. J Neurochem. 2016;139(1):11-21.

\section{Figures}




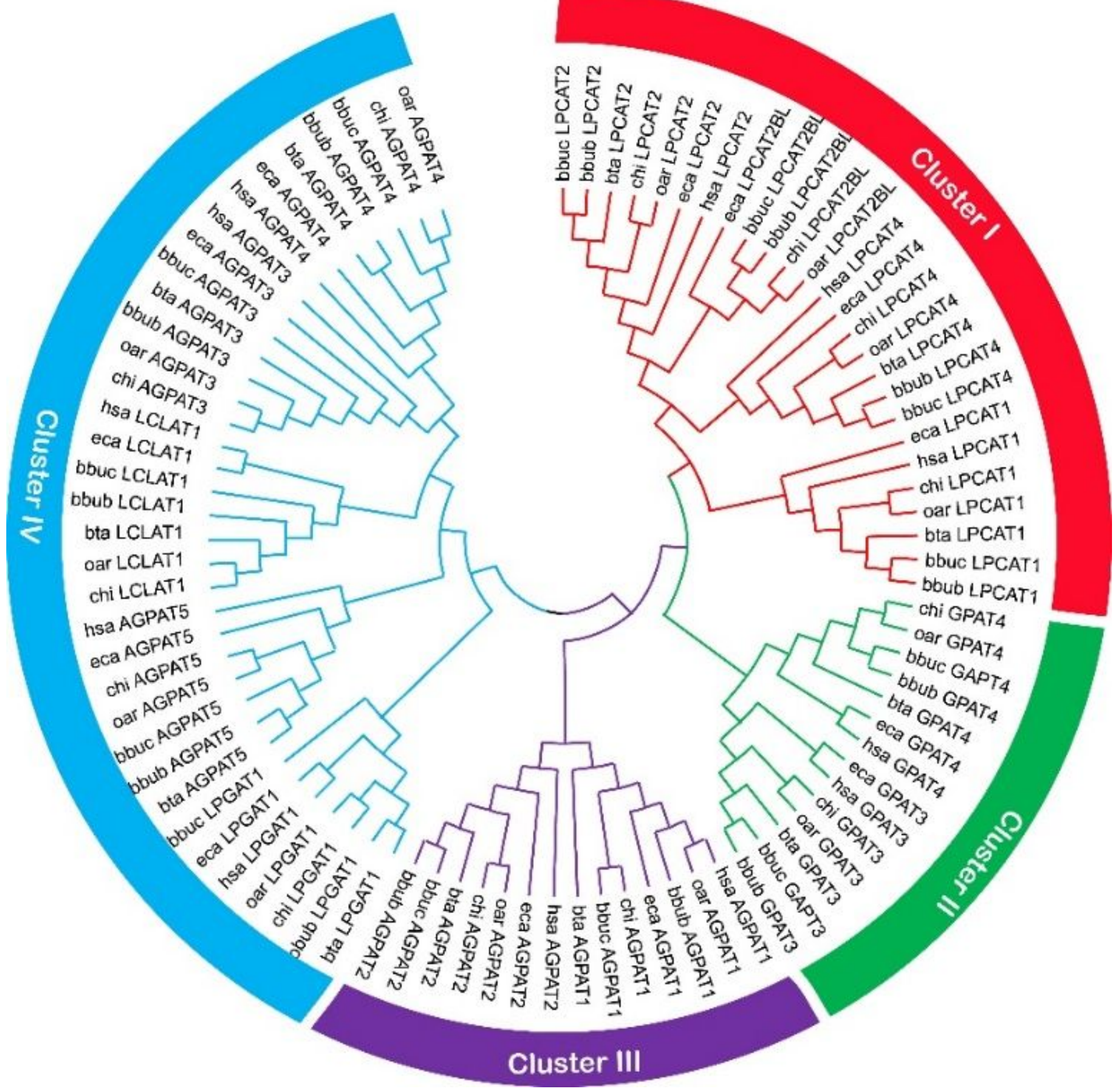

Figure 1

Phylogenetic relationship of the AGPAT proteins in seven representative mammals. Line or circle with different colors indicates different clusters. River buffalo: bbub; Swamp buffalo: bbuc; Cattle: bta; Goat: chi; Sheep: oar; Horse: eca; Human: hsa 


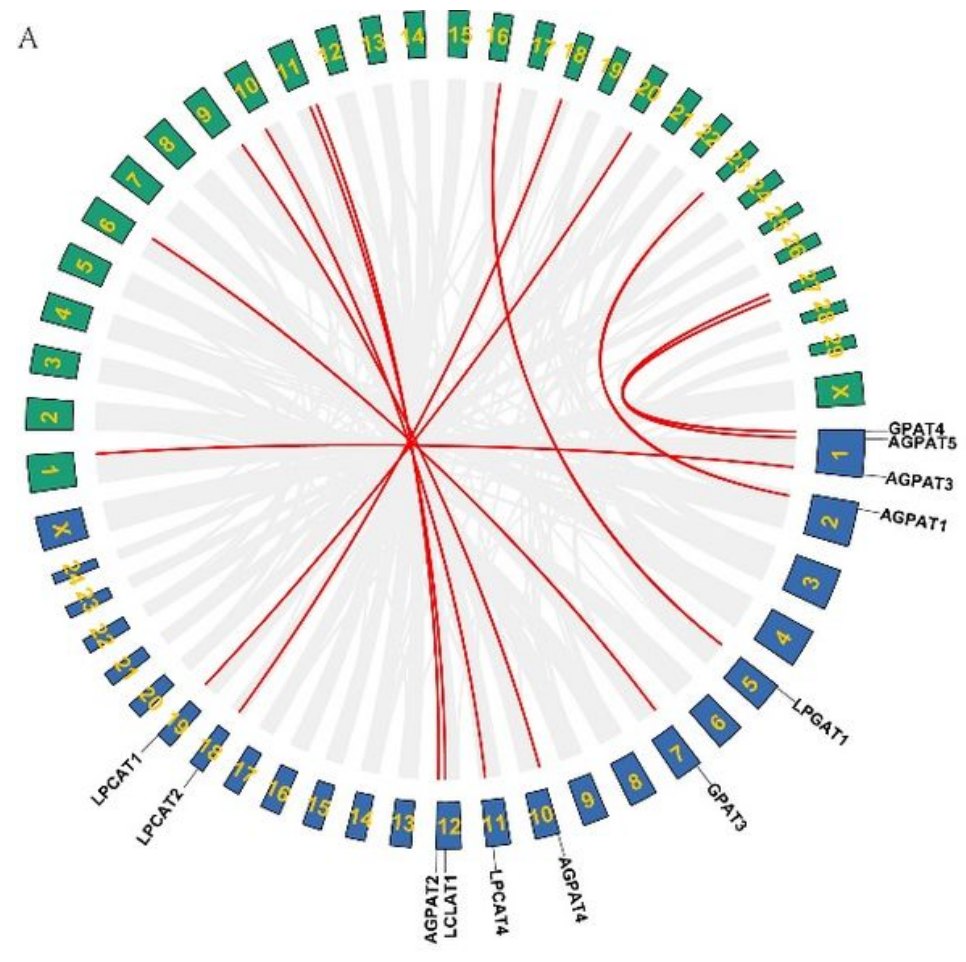

B

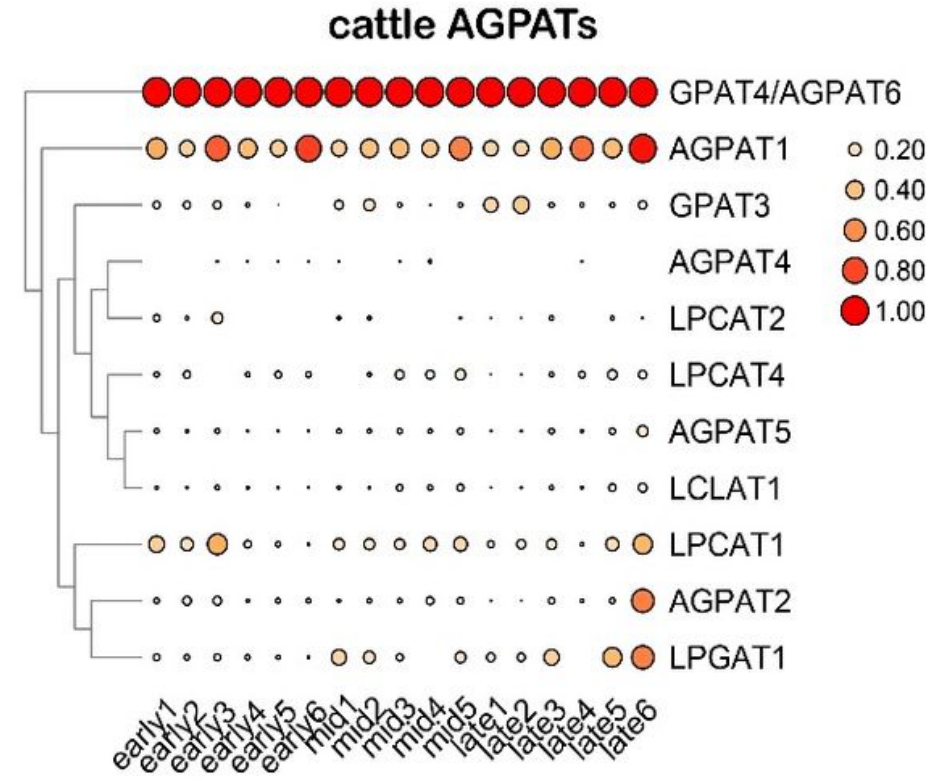

C

buffalo AGPATs

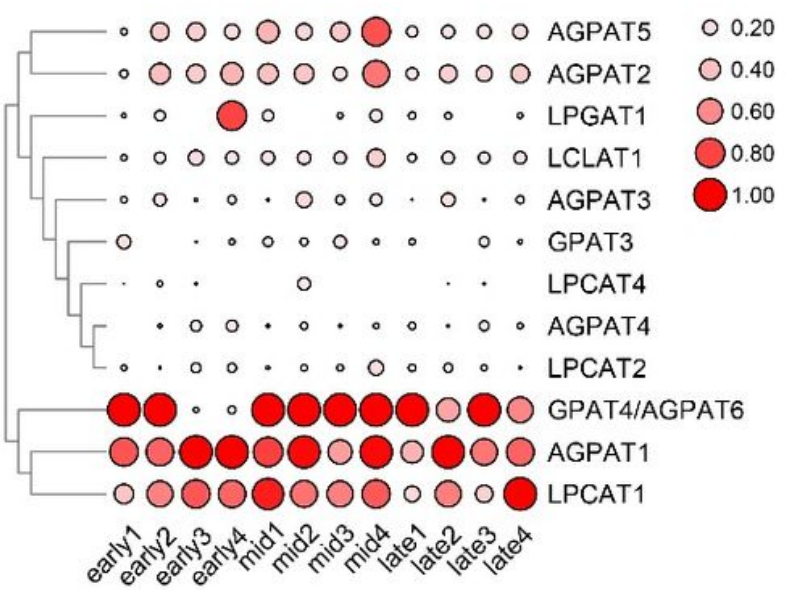

D

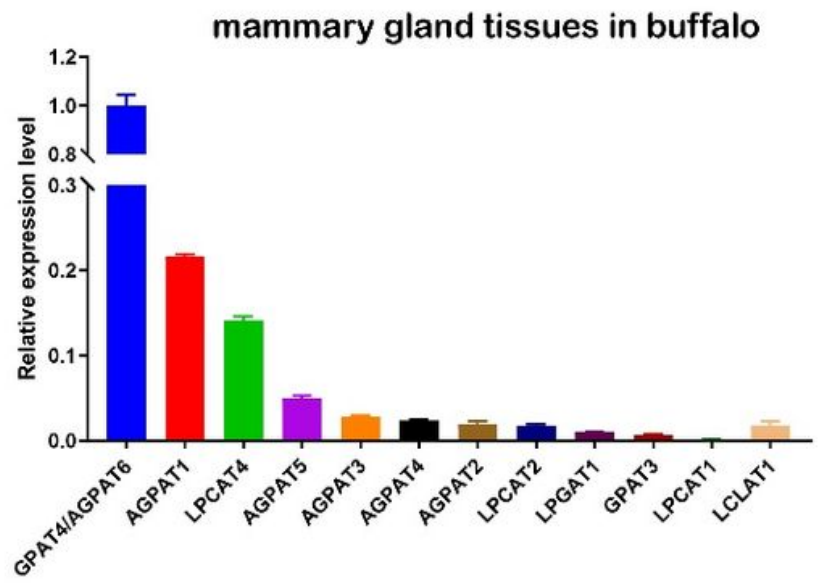

Figure 2

Collinearity and expression analysis of AGPAT family between buffalo and cattle. (A) Collinearity analysis of AGPAT family between buffalo and cattle. The green frame indicates the cattle chromosomes, the blue frame indicates the buffalo chromosomes, the red line represents the collinearity gene pairs. (B) Transcriptome analysis of cattle AGPAT family. (C) Transcriptome analysis of buffalo AGPAT family. (D) Expression analysis of buffalo AGPAT genes in mammary gland tissue. The area indicates the scale size. 
A

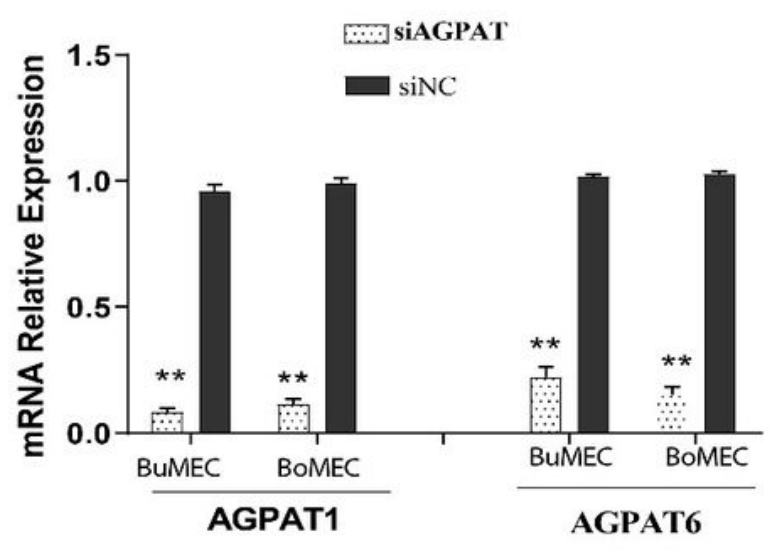

D

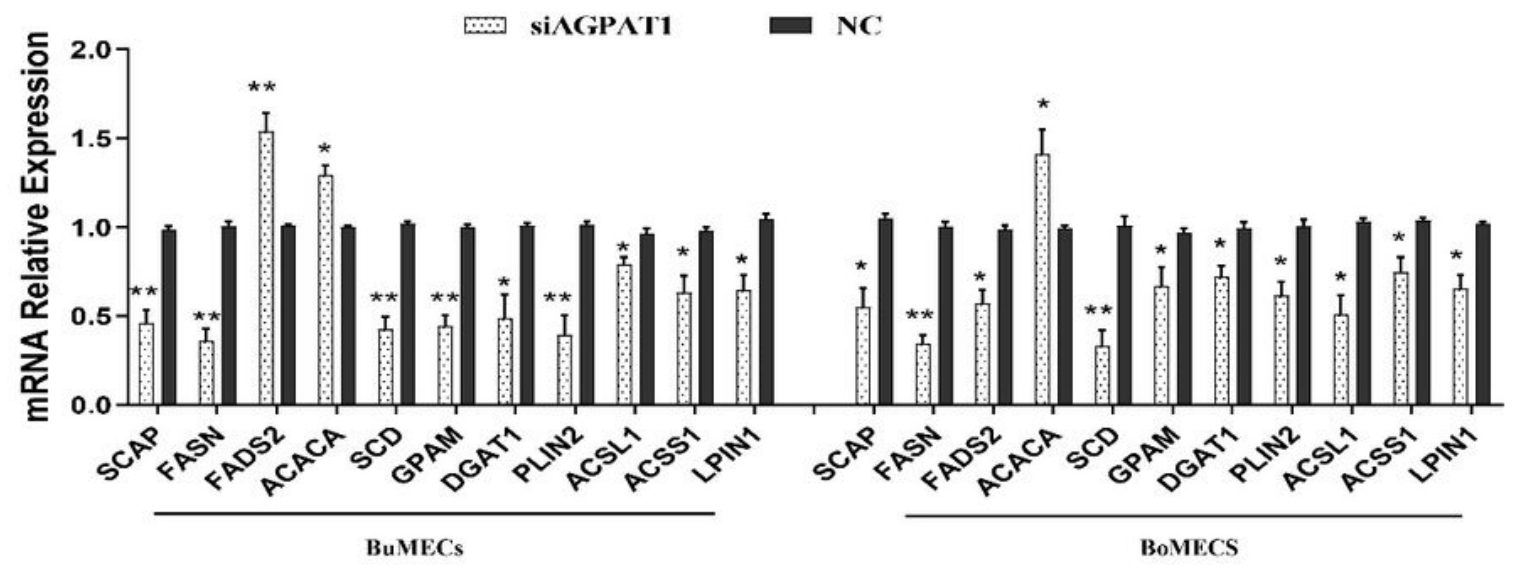

$\mathrm{E}$

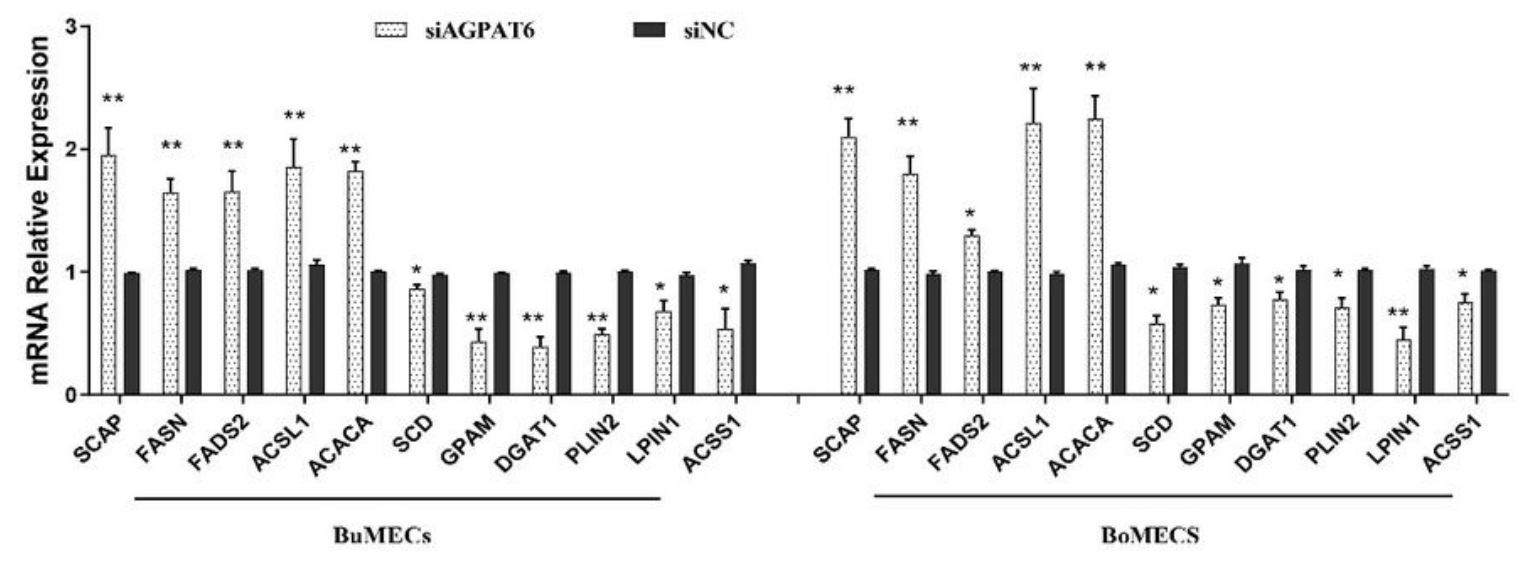

Figure 3

AGPAT1 and AGPAT6 regulated the TAG synthesis of BuMECs and BoMECs. (A) The interference efficiency analysis of AGPAT1 and AGPAT6 in BuMECs and BoMECs, respectively; (B) Effect of AGPAT1 knockdown on the TAG content of BuMEC and BoMECs; (C) Effect of AGPAT6 knockdown on the TAG content of BuMEC and BoMECs; (D) Effect of AGPAT1 knockdown on the expression of genes related to the TAG synthesis in BuMEC and BoMECs, respectively; (E) Effect of AGPAT6 knockdown on the 
expression of genes related to the TAG synthesis in BuMEC and BoMECs, respectively. ${ }^{*}<<0.05$; ${ }^{\star *} \mathrm{P}<$ 0.01 .

A

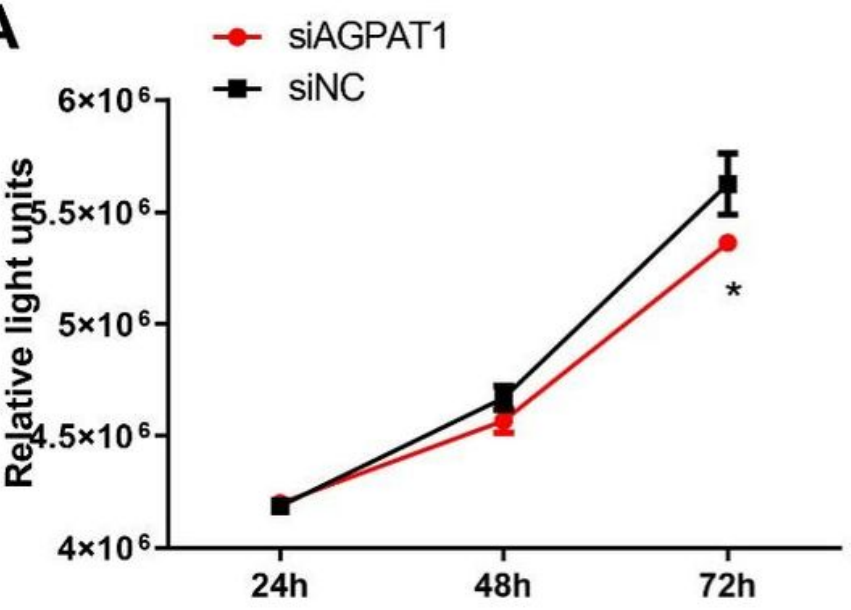

C

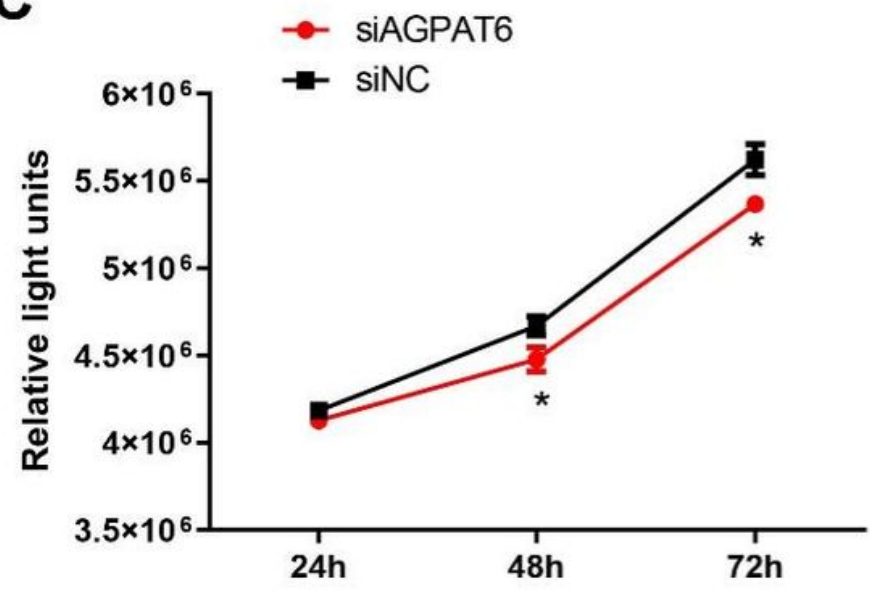

B

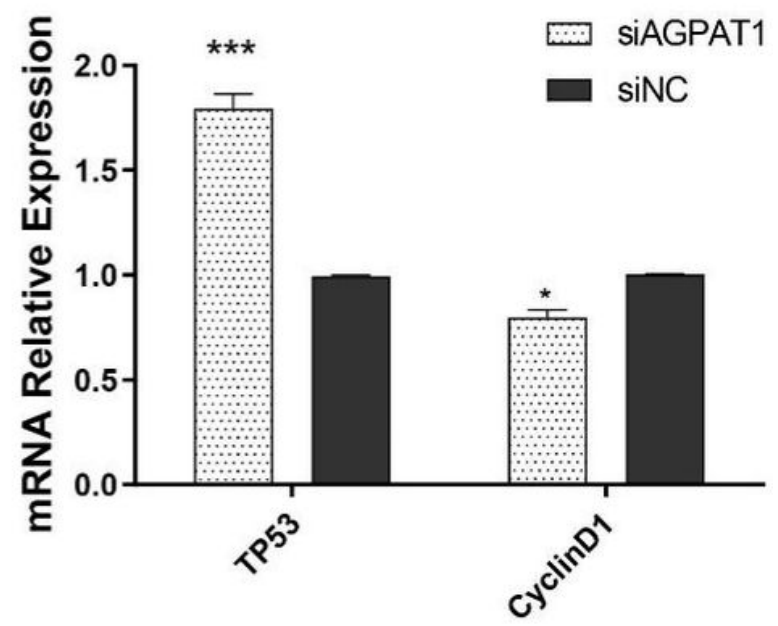

D

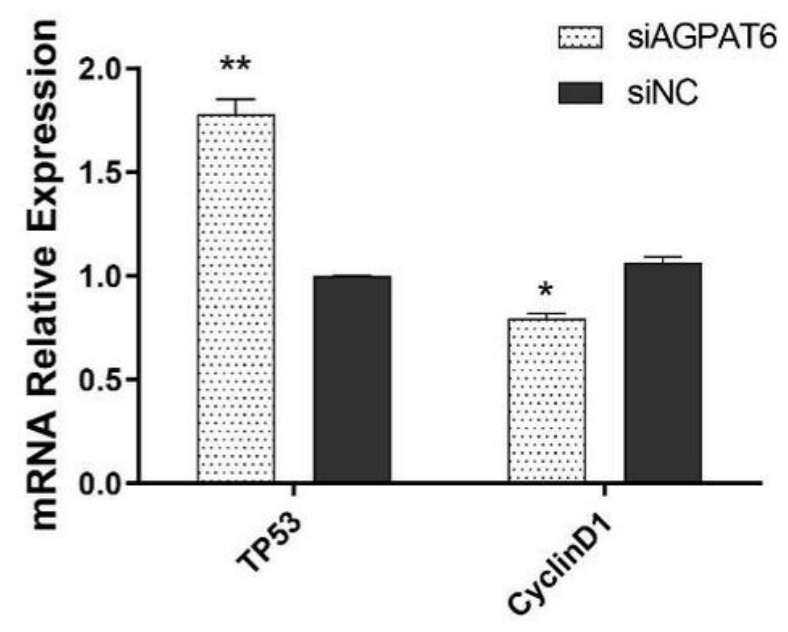

Figure 4

AGPAT1 and AGPAT6 induced cell proliferation of BuMECs. (A) Effect of AGPAT1 knockdown on BuMECs by the CCK-8 detection; (B) Effect of AGPAT1 knockdown on proliferation-related gene expression; (C) Effect of AGPAT6 knockdown on BuMECs by the CCK-8 detection; (D) Effect of AGPAT6 knockdown on proliferation-related genes expression. ${ }^{\star} P<0.05$; ${ }^{\star} \mathrm{P}<0.01$. 
A

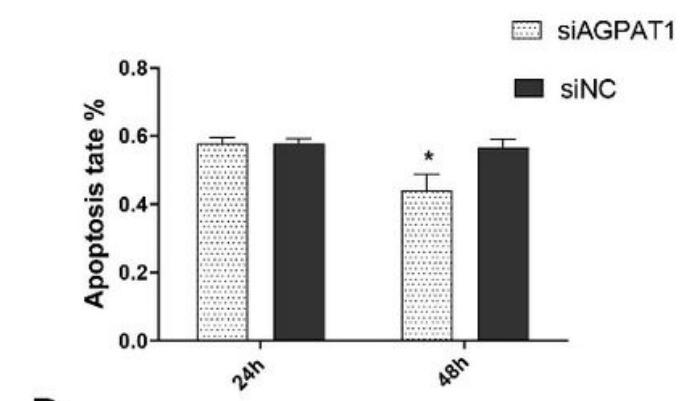

D

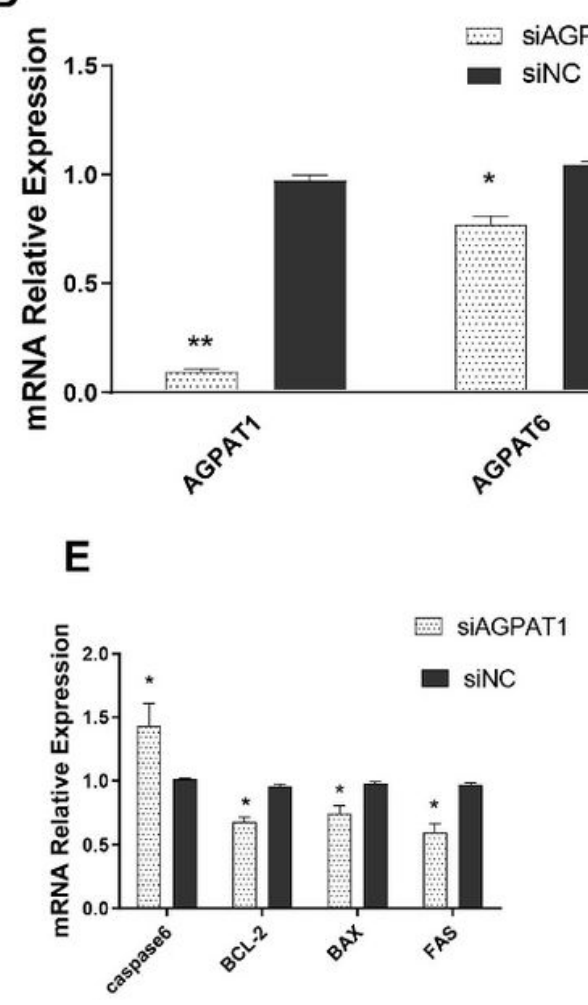

B

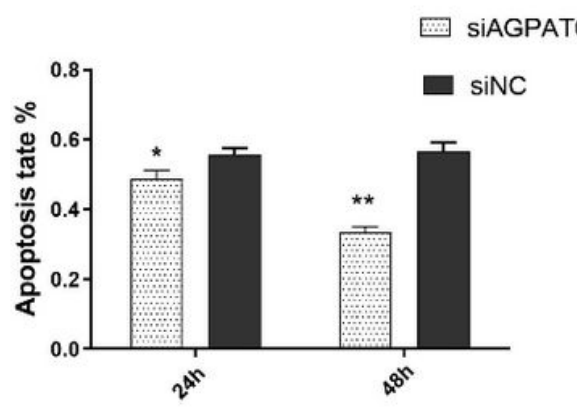

siAGPAT6

- siNC
C SIAGPAT1+SIAGPAT6 - sinc

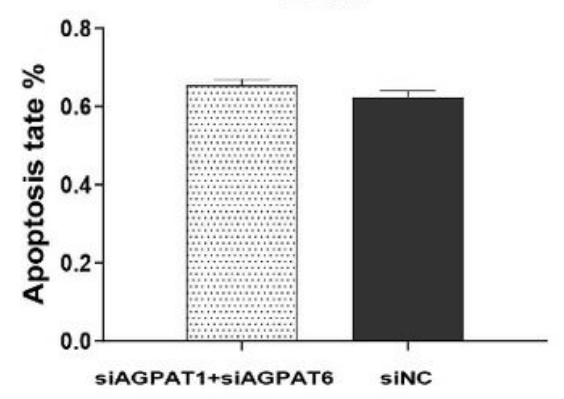

SIAGPAT1+SIAGPAT6

- si-NC

AGPAT1

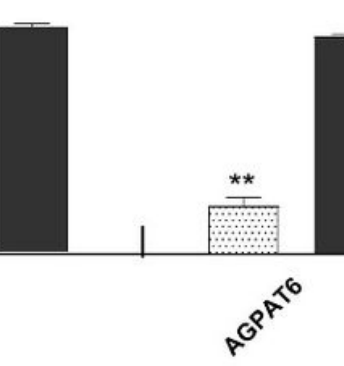

F

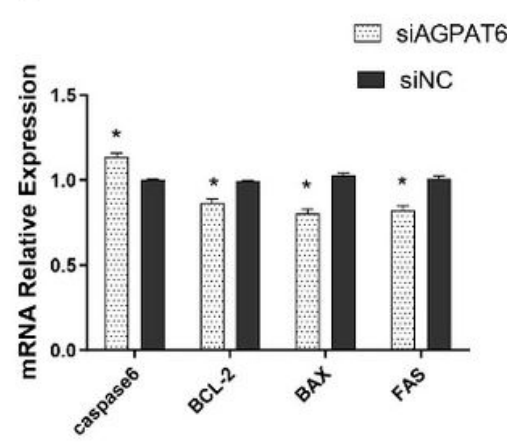

siAGPAT1+siAGPAT6

- siNC

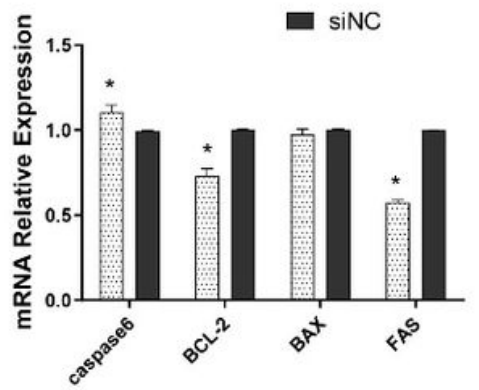

Figure 5

AGPAT1 and AGPAT6 promote cell apoptosis of BuMECs. (A) Apoptosis rates detection of AGPAT1 knockdown in BuMECs; (B) Apoptosis rates detection of AGPAT6 knockdown in BuMECs; (C) Apoptosis rates detection of both AGPAT1 and AGPAT6 knockdown in BuMECs; (D) The expression levels of AGPAT1 and AGPAT6 after AGPAT1 or/and AGPAT6 silencing at 48 h; (E) Effect of AGPAT1 knockdown on apoptosis-related genes expression; (F) Effect of AGPAT6 knockdown on apoptosis-related genes expression; (G) Effect of both AGPA1 and AGPAT6 knockdown on apoptosis-related genes expression. *P $<0.05 ;{ }^{*} \mathrm{P}<0.01$.

\section{Supplementary Files}

This is a list of supplementary files associated with this preprint. Click to download.

- FigureS1.jpg

- FigureS2.jpg 
- Tables1.xlsx

- Tables2.xIsx

- TableS3.xlsx

- Tables4.xlsx

- TableS5.xlsx 